\title{
thelomj
}

Letters QRISK validation and evaluation

\section{Bespoke cohort studies needed}

BMJ 2009; 339 doi: http://dx.doi.org/10.1136/bmj.b3512 (Published 01 September 2009) Cite this as:

BMJ 2009;339:b3512

Richard Morris, professor of medical statistics and epidemiology1, Irene Petersen, MRC training fellow1, Louise Marston, research statistician1, Kate Walters, clinical senior lecturer1, James Carpenter, reader in medical and social statistics ${ }^{2}$, Irwin Nazareth, professor of primary care 1 ${ }^{1}$ Department of Primary Care and Population Health, University College London, Royal Free Campus, London NW3 2PF ${ }^{2}$ Medical Statistics Unit, London School of Hygiene and Tropical Medicine, London WC1E 7HT

r.morris@pcps.ucl.ac.uk

Despite Collins and Altman's re-analysis of data from the THIN database to validate the QRISK equation for predicting cardiovascular disease, 1 adoption of QRISK in primary care is premature because key issues about the handling of missing data and the use of social deprivation indices remain unresolved.

Collins and Altman again highlight that complete data were available for just over a quarter of subjects. We appreciate that imputation methods were applied, but we question use of age-sex means of QRESEARCH data for lipid concentrations and blood pressures. This implies that QRESEARCH data were missing completely at random within age-sex strata-an assumption acknowledged as incorrect when the developers of QRISK revised their equation2. It also implies that observed QRESEARCH data reflect age-sex norms in the population-an assumption questioned by the developers' comparison of their data with the health survey for England. 3 We call for additional validation using data from bespoke cohort studies in which much greater attention has been paid to completeness of data.

Deprivation indices by their nature quickly become outdated, the Townsend index in particular being based on data from the 2001 census. It should be replaced with variables whose meaning is less context dependent, and which reflect underlying causes of inequalities in cardiovascular disease. For example, subcategorising non-smokers as either "ex-smokers" or "never smokers" would perhaps diminish some of the apparent predictive power of the Townsend index (since former smoking is likely to be more common among more deprived communities) and would allow QRISK to be more portable in its future use.

\section{Notes}

Cite this as: $B M J$ 2009;339:b3512

\section{Footnotes}

- Competing interests: None declared. 


\section{References}

1. Collins GS, Altman DG. An independent external validation and evaluation of QRISK cardiovascular risk prediction: a prospective open cohort study. BMJ2009;339:b2584. (7 July.)

2. Hippisley-Cox J, Coupland C, Vinogradova Y, Robson J, May M, Brindle P. QRISK: authors' response. bmj.com 2007. www.bmj.com/cgi/eletters/335/7611/136\#174181

3. Hippisley-Cox J, Coupland C, Vinogradova Y, Robson J, May M, Brindle P. Derivation and validation of QRISK, a new cardiovascular disease risk score for the United Kingdom: prospective open cohort study. BMJ2007;335:136. 